\title{
Frictional and bone ingrowth properties of engineered surface topographies produced by electron beam technology
}

\author{
J. Elizabeth Biemond · René Aquarius • \\ Nico Verdonschot $\cdot$ Pieter Buma
}

Received: 26 March 2010 / Published online: 16 December 2010

(C) The Author(s) 2010. This article is published with open access at Springerlink.com

\begin{abstract}
Background Electron beam melting (E-beam) is a new technology to produce 3-dimensional surface topographies for cementless orthopedic implants.

Methods The friction coefficients of two newly developed E-beam produced surface topographies were in vitro compared with sandblasted E-beam and titanium plasma sprayed controls. Bone ingrowth (direct bone-implant contact) was determined by implanting the samples in the femoral condyles of 6 goats for a period of 6 weeks.

Results Friction coefficients of the new structures were comparable to the titanium plasma sprayed control. The direct bone-implant contact was 23.9 and $24.5 \%$ for the new surface structures. Bone-implant contact of the sandblasted and titanium plasma sprayed control was 18.2 and $25.5 \%$, respectively.

Conclusions The frictional and bone ingrowth properties of the E-beam produced surface structures are similar to the plasma-sprayed control. However, since the maximal bone ingrowth had not been reached for the E-beam structures during the relatively short-term period, longer-term followup studies are needed to assess whether the E-beam struc-
\end{abstract}

J. E. Biemond $(\varangle) \cdot$ R. Aquarius $\cdot$ N. Verdonschot · P. Buma Orthopaedic Research Laboratory,

Radboud University Nijmegen Medical Center, PO Box 9101, 6500 HB Nijmegen, The Netherlands e-mail: L.Biemond@orthop.umcn.nl

N. Verdonschot

Laboratory for Biomechanical Engineering,

University of Twente, PO Box 217,

7500 AE Enschede, The Netherlands tures lead to a better long-term performance than surfaces currently in use, such as titanium plasma spray coating.

Keywords Electron beam melting - Bone ingrowth . Friction $\cdot$ Surface characteristics $\cdot$ Prosthesis

\section{Introduction}

Cementless fixation is used in about two-thirds of all primary total hip replacements (THR) in the United States [1]. Although the overall results of THR are good, $5-10 \%$ of the cementless implants still fail within 10 years of implantation $[2,3]$. Instability, aseptic loosening and infection are the most important reasons for implant failure within 5 years of implantation, whereas aseptic loosening is by far the main reason for failure after 5 years [4]. The etiology of aseptic loosening is multifactorial: wear debris, stress shielding and micromotion at the bone-implant interface play a role [5]. The frequency of failure is likely to increase due to the implantation in younger and more active patients [6]. Revision surgery after failure of an implant creates large burdens for both the patient and society [7]; thus, there is a need for improvement of cementless implants in order to avoid aseptic loosening. Although the process of aseptic loosening is not completely understood [2], roentgen stereo-photogrammetric analysis studies have shown that long-term loosening rates after a mean follow-up period of 8 years were highly correlated with early postoperative migration [8]. This strongly suggests that inadequate direct postoperative bone-implant interface characteristics play a crucial role in the fate of the implant behavior and its survival. A high friction coefficient between the implant and the bone will enhance initial stability with low micromotions at the interface [9]. Sufficient frictional 
stability will enable bone ingrowth into the surface of the implant, resulting in a long lasting mechanical interlock of the implant and optimal secondary mechanical stability.

In this light, a new implant should be designed not only with respect to mechanical properties (mechanical strength and elastic modulus of the bulk material) and surface chemistry, but frictional and bone ingrowth properties should be taken in account as well in order to avoid implant failure [10]. This can be achieved by selecting the optimal combination of surface roughness, pore size and porosity of the ingrowth layer [11]. With respect to initial stability, it has been demonstrated that an increased roughness creates more interface friction in addition to an increase in bone ingrowth (measured by bone-implant contact) $[12,13]$. With respect to secondary stability, coatings with porosities of 50\% show good survival [14]; furthermore, a substantial increase in fixation strength can be obtained by increasing the porosity of implants to $75-80 \%$ [15]. It is necessary to have interconnectivity between the pores in order to permit bone ingrowth [16]. There is, however, an upper limit in the porosity due to the restrictions associated with mechanical strength properties [17]. In general, a high porosity is related to an increased pore size. With respect to pore size, a diameter of at least $100 \mu \mathrm{m}$ is needed to allow bone ingrowth and eventually vascularisation [18]. Although the optimal pore size has yet to be determined, it is evident that this parameter too affects bone ingrowth [17].

The aforementioned surface characteristics of cementless implants can be modified by various techniques, such as plasma spraying or sintering of metal powder and fibers. A particularly promising and relatively new technology to produce new surface geometries is electron beam melting (E-beam) [19-22]. Based on rapid prototyping technology, implants are built up from metal powder in a 'layer-bylayer' fashion. Each layer is melted by electron beam exposure to reproduce the geometry defined by a 3D CAD model. The E-beam technology enables the production of specimens with both solid and porous zones, which makes it possible to produce a solid implant with a porous surface structure in one manufacturing step [21].

For this study two new E-beam engineered surface topographies were produced. It was hypothesized these two structures referred to as the 'wave' and 'cubic' topographies would provide for both high frictional properties and adequate bone ingrowth characteristics.

This resulted in the following research questions: (1) Do the new surface structures provide enough friction at the bone-implant interface in order to achieve initial stability? (2) Do the new surface structures allow good bone ingrowth compared to conventionally made implant surfaces?

\section{Materials and methods}

Implants

The implants were made out of Ti6Al4V powder and produced with E-beam technology (Eurocoating SpA, Trento, Italy). In total 28 flat specimens were produced for the friction experiment and 24 cylindrical specimens for the in vivo experiment. The powder size used in the E-beam process ranged from 45 to $100 \mu \mathrm{m}$. The E-beam specimens were created using a 3D CAD model which was segmented into layers of $0.1 \mathrm{~mm}$ in order to generate layer information. Subsequently, a homogeneous powder layer was applied on the process platform in a vacuum chamber at constant high temperature $\left( \pm 700^{\circ} \mathrm{C}\right)$. The electron beam scanned the powder layer line by line and melted the loose powder particles at programmed locations forming a compact layer in the desired shape. The process platform was then lowered by one layer thickness $(0.1 \mathrm{~mm})$ and a new powder layer (of $0.1 \mathrm{~mm}$ thickness) was applied after which the process is repeated $[19,20]$. Upon completion, all specimens were sandblasted with corundum and cleaned in a specific washer for medical devices. Subsequently, the specimens are dried, packed and steam sterilized in an autoclave. The overall accuracy of the E-beam technology in terms of computer model reconstruction, and thereby the tolerance of the E-beam specimens, is $\pm 0.15 \mathrm{~mm}$. The adhesive strength (test described in ASTM F1147) of the specimens was $>50 \mathrm{MPa}$ and the Taber abrasion test (as described in ASTM F1978) showed a weight loss after 100 cycles of $44.00 \pm 8.42 \mathrm{mg}$ for the wave and $27.78 \pm$ $4.51 \mathrm{mg}$ for the cubic structure.

Four different specimens were made with the E-beam technique. Two experimental porous specimens, each with a unique surface topography (either "wave" or "cubic", Fig. 1). And two solid, control specimens; one with a commercially available conventional titanium plasma spray coating (TiPore, thickness $350 \mu \mathrm{m}$ ) [23] and one that was only sandblasted in order to remove particulate debris) (Fig. 1). To characterize the topographic surface structures, the specimens were embedded in methylmethacrylate (MMA) and analyzed by light microscopy (Axioplan 2, Zeiss, Oberkochen, Deutschland). Surface roughness values of the specimens were determined using a Universal Surface Tester (UST) (Innowep, Wurzburg, Germany).

\section{Friction experiment}

Friction at the bone-implant interface of the two new surface structures and the two control surfaces was evaluated using seven specimens from each group. The surface structure was applied on one side of the flat specimen $(7 \times 55 \times 4 \mathrm{~mm})$, whereas the other side was polished. 


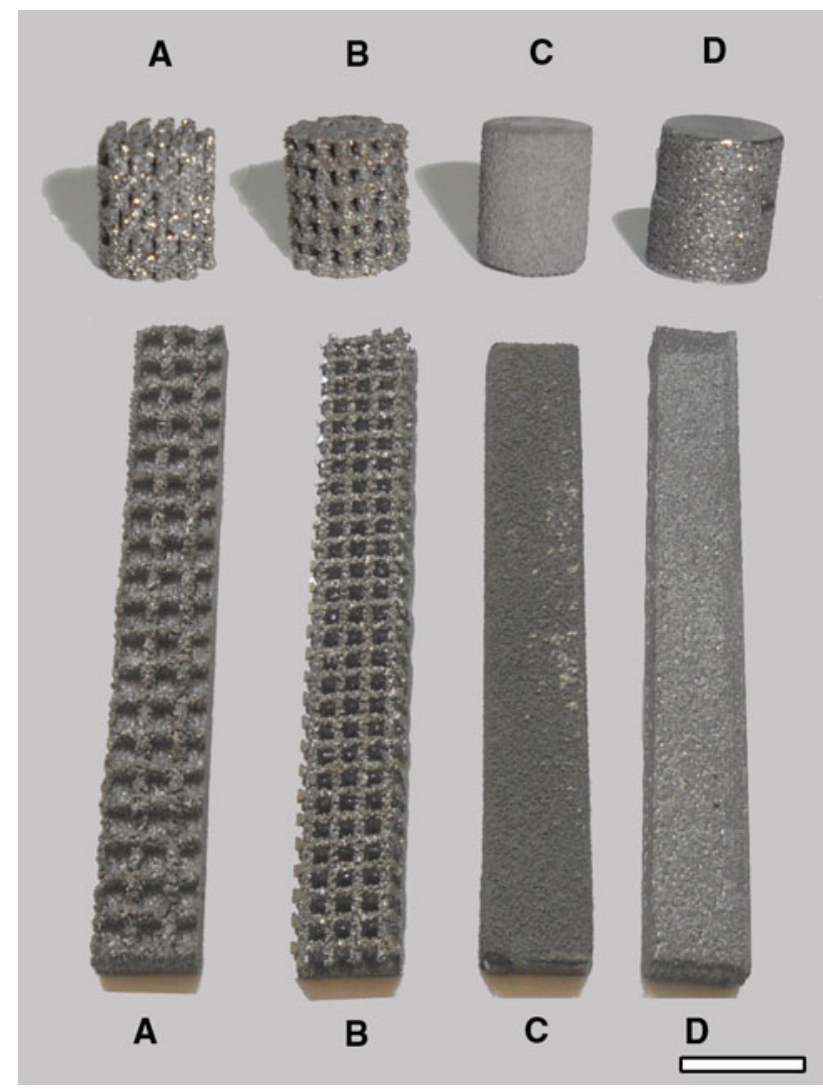

Fig. 1 Specimens. On top the specimens for the in vivo experiment, below the specimens for the friction test. $A$ wave structure, $B$ cubic structure, $C$ plasma spray coating and $D$ sandblasted surface. (bar $10 \mathrm{~mm}$ )

A complete femur was obtained from a fresh frozen human cadaver provided by the Anatomy department of the Radboud University Nijmegen Medical Center according to
Dutch law. Box-shaped bone samples $(12 \times 20 \times 4 \mathrm{~mm})$ were machined from the cortical bone of this femur. A DEXA scan (QDR 4500, Hologic Inc., Bedfort, MA, USA) was performed to define the bone mineral density of each bone sample (average $\mathrm{BMD}=0.371 \mathrm{~g} / \mathrm{cm}^{2}, \mathrm{SD}=$ $0.047 \mathrm{~g} / \mathrm{cm}^{2}$ ). Subsequently each specimen was assigned to a specific bone sample, randomizing for BMD. The bone sample was securely placed in a jig and the specimen was positioned against the bone sample, clamping it with a lowfriction roller bearing. Subsequently, this setup was placed in a $37^{\circ} \mathrm{C}$ water basin and stabilized by a counterweight providing a normal force (Fn, $40 \mathrm{~N}$ ) (Fig. 2).

A material testing system (MTS 458.2 MicroConsole ${ }^{\mathrm{TM}}$, Minneapolis, MN, USA) was used to rub the titanium specimens alongside the bone specimen with a fixed displacement rate $(0.33 \mathrm{~mm} / \mathrm{s})$ over a distance of $30 \mathrm{~mm}$. The required force was measured and divided by the normal force in order to define the friction coefficient.

\section{In vivo experiment}

Surgery was performed on six (sample size calculation: power $=0.8, \alpha=0.05$ ) female, skeletal mature goats (Capra Hircus Sana), weighing 47-72 kg (mean 55). Each goat received four different cylindrical implants $(\varnothing 8 \mathrm{~mm}$, length $10 \mathrm{~mm}$ ), two with the new topographic structures and two controls. Three different implantation areas on each of the hind legs were used: the lateral femoral condyle, the medial femoral condyle and the region dorsal of the femoral trochlea. The used model was combined out of two existing models $[24,25]$ in order to maximize the amount of specimens in each goat. The new and control surface structures
Fig. 2 Friction test apparatus. A picture and schematic drawing of the friction apparatus. A roller bearing $(R)$ was used to position the specimen $(S)$ on the bone sample $(B)$. A normal force $(F n)$ was used to stabilize the specimen on the bone. The specimen was pushed alongside the bone sample by the MTS (Fmts)
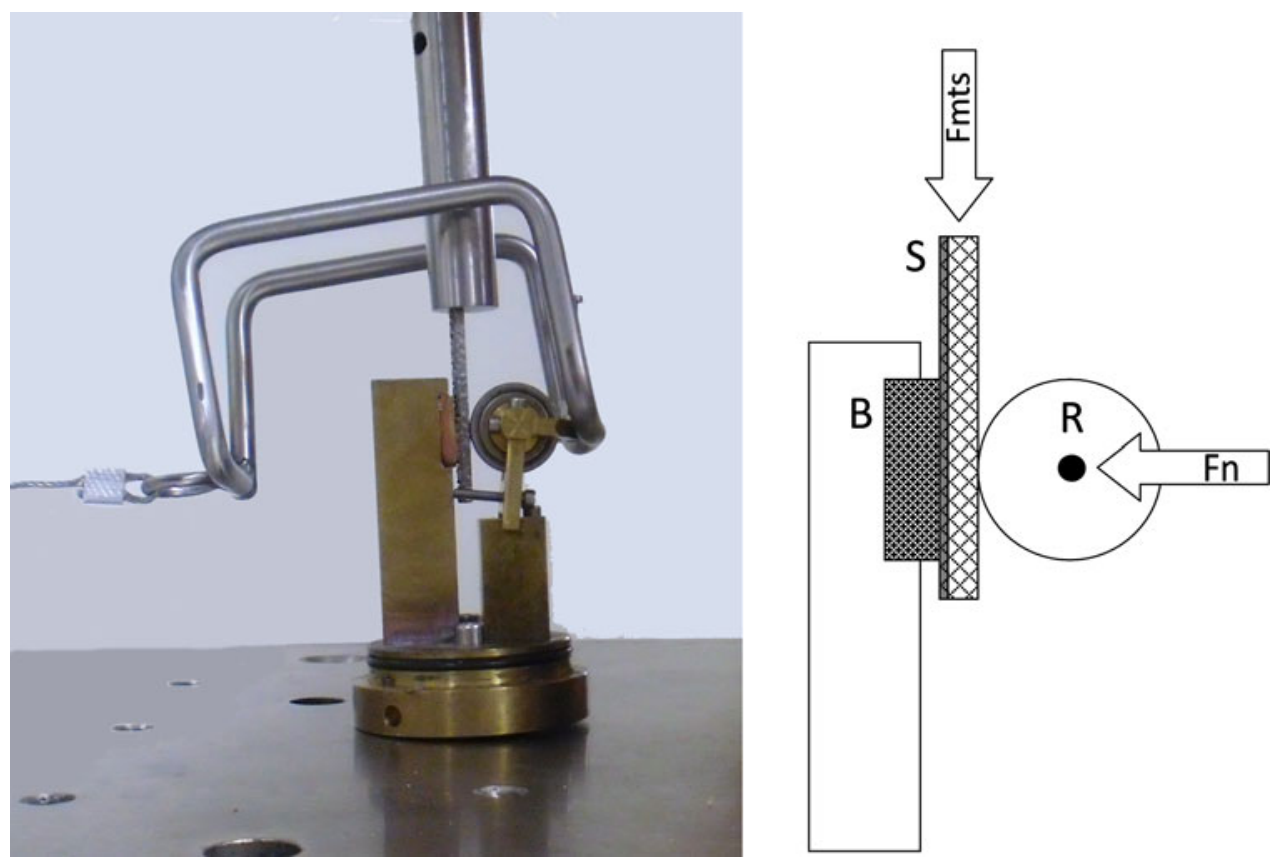
were equally divided among the implantation areas, by which two out of six implantation areas in each goat were not used.

The goats were anesthetized with propofol $(4 \mathrm{mg} / \mathrm{kg}, \mathrm{B}$. Brown, Melsungen, Germany), intubated and anesthesia was maintained using isoflurane. The goats were placed in a supine position and the implantation procedure was performed under strict sterile conditions. The knee was approached medially, visualizing the origin of the medial collateral ligament. Just proximal to the origin a hole was drilled into the medial condyle. Sharp cannulated drills with an increasing diameter $(\varnothing 6.0$ and $8.0 \mathrm{~mm}$ ) were used. Saline was used during the drilling to prevent heat-induced necrosis. The hole was inspected to guarantee the specimen would be completely surrounded by trabecular bone. The implant was inserted into the hole and the facia and skin were closed separately with resorbable sutures. This procedure was repeated on the lateral condyle and a third area located proximal to the hole in the lateral condyle, perpendicular to the cortex. In this way the specimen was implanted dorsal of the femoral trochlea. The goats received postoperative ampicillin $(7.5 \mathrm{mg} / \mathrm{kg}$ Intervet, Boxmeer, The Netherlands) for 4 days and were housed at a farm. Fluorochromes were administered by subcutaneous injection at 2 (tetracyclin), 4 (xylenol orange) and 6 weeks (calcein green) after surgery during 2 days in order to make it possible to assess bone ingrowth at these time points. Goats were killed 6 weeks and 2 days postoperative by an overdose of sodium pentobarbital (Euthesate, Ceva Santa Animale, Libourne, France). This study was approved by the animal ethics committee of the Radboud University Nijmegen and the NIH principles of laboratory animal care were followed.

Histological analysis

After sacrificing the animals, the distal femurs were retrieved. Each specimen and the surrounding bone tissue was fixed in phosphate-buffered $4 \%$ formaldehyde solution for 4 days and embedded in MMA. Slices of ca. $40 \mu \mathrm{m}$, perpendicular to the length of the specimen, were cut using a sawing microtome (SP 1600, Leitz, Wetzlar, Germany). Quantitative analysis of bone ingrowth was performed using fluorescence microscopy on unstained slices and light microscopy on hematoxylin/eosin (HE) stained slices.

Each slice was analyzed using AnalySIS (AnalySIS 3.2 Soft Imaging System, Münster, Germany). For the bone ingrowth depth measurement, the sectioned specimen was divided into 4 quadrants and bone ingrowth depth after 4 and 6 weeks was measured in each quadrant. The ingrowth depth used for analysis was defined as the average of the maximum ingrowth depth for bone (distance from the outline of the specimen to the deepest fluorochrome label, Fig. 3) in each of these quadrants. The surface areas of bone and pores inside the specimen were measured. Subsequently, the percentage of bone (bone area \%) in the porous area of the specimen was calculated by bone area/porous area. It was not possible to measure ingrowth depth and bone area $\%$ for the sandblasted and the plasma sprayed coated specimens, due to the solid structure of these control specimens.

Direct contact between bone and the specimen inside the pores was projected on the circumference of the implant. The percentage of direct bone-implant contact was defined as the sum of these projections divided by the circumference of the specimen (Fig. 3).

\section{Statistical analyses}

An unpaired $t$ test was used to compare the friction coefficients of the new surface structures with the controls. SPSS (16.0, SPSS Inc., Chicago, USA) was used to perform a multivariate regression analysis of friction, with surface structure and BMD as predictor variables.
Fig. 3 Measurement method for bone ingrowth depth and direct bone-implant contact. The arrow represents the maximum bone ingrowth depth of this quadrant. Direct bone-implant contact was projected onto a circle representing the circumference of the implant in order to determine the percentage of direct bone-implant contact
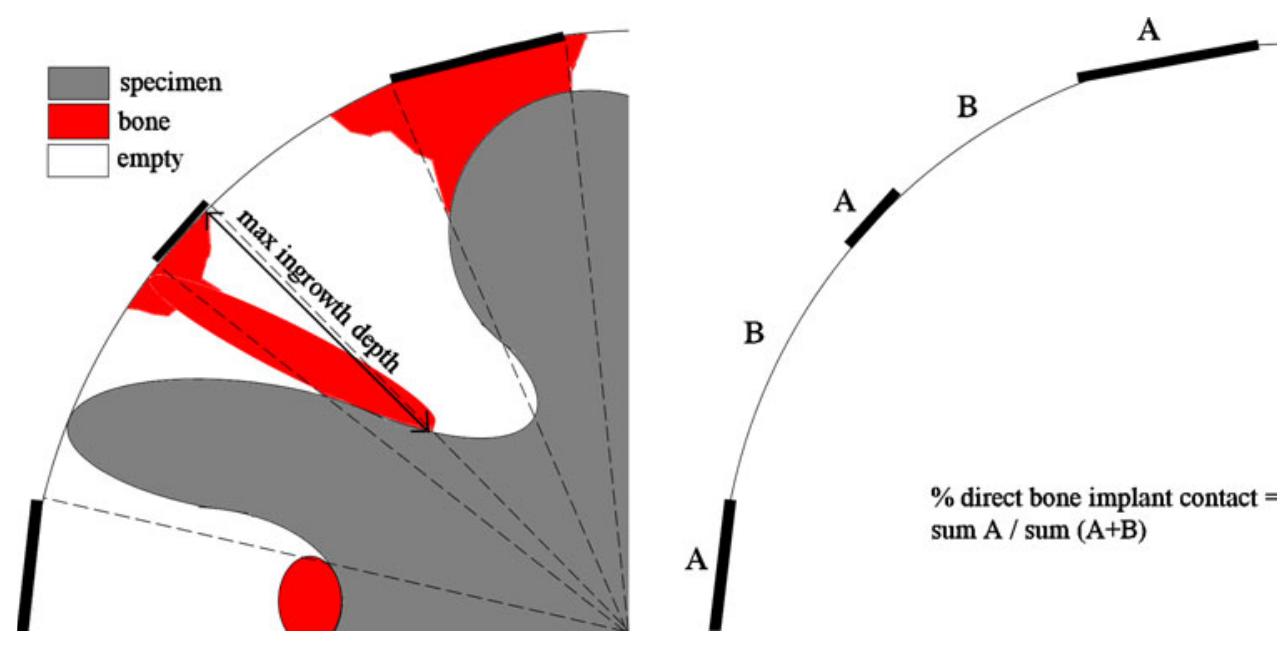
Statistical analysis of the in vivo experiment was performed using a Mann-Whitney $U$ test for both ingrowth depth and bone area percentage. A univariate analysis of variance of the ranked results was performed for direct bone-implant contact using the following fixed factors: surface structure, implantation area and goat. Statistical significance was set at $p<0.05$.

\section{Results}

\section{Implant characterization}

Analysis of the embedded specimens by light microscopy showed that the wave and cubic structures had average pore sizes of 0.9 and $1.2 \mathrm{~mm}$ and porosities of 49 an $77 \%$, respectively. The nominal thickness of the porous layer was 1.35 and $1.95 \mathrm{~mm}$ for the wave and cubic structure, respectively.

The results of the roughness measurements showed an average surface roughness $(\mathrm{Ra})$ of 6.34 for the wave structure, 5.22 for the cubic structure, 6.09 for the titanium plasma sprayed surface and 3.52 for the sandblasted surface.

\section{Friction experiment}

The average friction coefficient was $0.68(\mathrm{SD}=0.04)$ for the wave structure and 0.63 ( $\mathrm{SD}=0.03$ ) for the cubic structure. The average friction coefficient of the controls was $0.64(\mathrm{SD}=0.04)$ and $0.49(\mathrm{SD}=0.06)$ for the titanium plasma sprayed and the sandblasted specimen, respectively. The friction coefficient of the sandblasted specimen was significantly $(p<0.001)$ lower than the new surface structures and the titanium plasma sprayed control (Table 1).

The multivariate regression analysis showed that the variation in BMD had no significant influence on the frictional properties of the surface structures.

In vivo experiment

\section{Clinical evaluation}

No intraoperative complications occurred during surgery. All goats were fully weight bearing within 1 week after surgery. Swelling of the knee was seen in three goats, without causing general illness of the goats. This indicates that there was no infection, which was confirmed by postmortem tissue cultures beside the implantation locations.

\section{Histology}

The HE-stained slices showed bone ingrowth into the pores of the new surface structures (Fig. 4). The results for ingrowth depth at 4 and 6 weeks (as measured using the xylenol orange and calceine green fluorochrome labeling, respectively), bone area percentage and direct boneimplant contact (both measured on HE-stained slices) are listed in Table 1.

The bone ingrowth depth of the cubic structure was greater compared to the wave structure at 4 and 6 weeks after surgery (1.18 vs 0.78 and 1.47 vs $0.98 \mathrm{~mm}$, respectively; $p=0.009)$. Contrastingly, the wave structure showed better

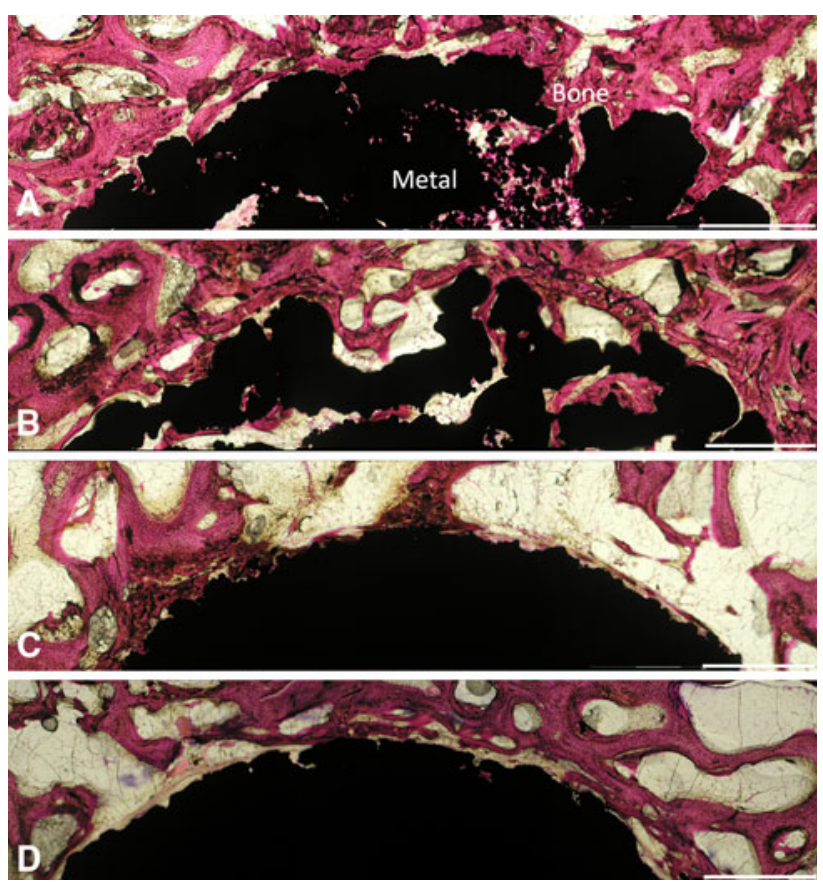

Fig. 4 Qualitative analysis of bone ingrowth. HE-stained slices of the wave (a) and cubic structure (b) with extensive bone ingrowth into the pores. Bone ingrowth on the titanium plasma sprayed (c) and sandblasted (d) control. (bar $1 \mathrm{~mm}$ )
Table 1 Friction coefficients $(n=7)$ and bone ingrowth characteristics $(n=6)$

\footnotetext{
Values are results and SD

$* p=<0.05$
}

\begin{tabular}{|c|c|c|c|c|c|}
\hline \multirow[t]{2}{*}{ Specimen } & \multirow{2}{*}{$\begin{array}{l}\text { Friction } \\
\text { coefficient }\end{array}$} & \multicolumn{2}{|c|}{ Ingrowth depth (mm) } & \multirow{2}{*}{$\begin{array}{l}\text { Bone } \\
\text { area }(\%)\end{array}$} & \multirow{2}{*}{$\begin{array}{l}\text { Bone implant } \\
\text { contact }(\%)\end{array}$} \\
\hline & & 4 weeks & 6 weeks & & \\
\hline Wave & $0.68(0.04)^{*}$ & $0.78(0.19)^{*}$ & $0.98(0.29)^{*}$ & $18.1(3.6)^{*}$ & $23.9(11.6)$ \\
\hline Cubic & $0.63(0.03)^{*}$ & $1.18(0.10)^{*}$ & $1.47(0.20)^{*}$ & $13.9(4.7)^{*}$ & $24.5(5.6)$ \\
\hline Ti-coated & $0.64(0.04)^{*}$ & NA & NA & NA & $25.5(13.3)$ \\
\hline Sandblasted & $0.49(0.06)^{*}$ & NA & NA & NA & $18.2(16.0)$ \\
\hline
\end{tabular}


results for bone area percentage than the cubic $(p=0.009)$ (Table 1).

The bone-implant contact of the new topographic surface structures was comparable to the titanium plasma sprayed control. No significant differences in bone-implant contact were observed throughout the four tested surfaces $(p=0.400)$, due to a large variation for the wave structure and the control surfaces (Table 1).

Statistical analysis showed that differences between the goats did not affect the bone-implant contact $(p=0.195)$. However, the location of the implantation had an effect on the bone-implant contact; it appeared that the direct bone implant contact was less in specimens implanted dorsally of the femoral trochlea relative to the two condyle areas $(p=0.027)$.

\section{Discussion}

Electron beam melting is a relatively new technique capable of producing complex 3-dimensional geometries [21]. With this technique many possible surface characteristics can be engineered thus aiding in the development of an optimal surface structure for bone ingrowth for cementless prostheses. In this study two controls were selected; a titanium plasma sprayed and a sandblasted specimen. The titanium plasma spray coating was selected as this coating is already widely used on femoral stems. Coated stems produce good medium- to long-term survival rates [23] and they perform better than uncoated cementless stems [26]. The plain sandblasted control was chosen to determine the effect of the E-beam technology itself.

Some limitations of the present study should be acknowledged. First of all, the frictional properties in contact with cortical bone were determined, whereas a prosthetic surface is also in contact with trabecular bone. The friction coefficient of a metal surface on cortical bone is generally lower than the same specimen on trabecular bone [27-29]. Therefore, the reported friction values are probably in the lower range of frictional values that occur around a hip prosthesis. Secondly, no chemical surface analysis was performed. Thirdly, the results for bone ingrowth showed a large variability, partly due to the rather large tolerance of the E-beam technology and differences in implantation location. Although not statistically significant it is likely that in between goat differences, overall biological variability and unavoidable differences in surgical precision (i.e. the quality of the press fit implantation) will affect this.

In this study the frictional properties of the surfaces were determined as these values have a direct effect on the stability potential of the metal surface relative to the bone surface. Obviously, the friction is influenced by the roughness of the metal structure and we considered quantifying the
Table 2 Frictional properties of coatings for orthopedic implants

\begin{tabular}{llll}
\hline Author & Coating & \multicolumn{2}{l}{ Friction coefficient } \\
\cline { 3 - 4 } & & $\begin{array}{l}\text { Cortical } \\
\text { bone }\end{array}$ & $\begin{array}{l}\text { Trabecular } \\
\text { bone }\end{array}$ \\
\hline Heiner [28] & $\begin{array}{l}\text { Sintered spherical } \\
\text { titanium beads }\end{array}$ & $0.63(0.10)$ & $1.18(0.39)$ \\
Fitzpatrick [27] & Porous tantalum & $0.74(0.07)$ & $0.88(0.09)$ \\
Zhang [29] & Porous tantalum & $0.74(0.07)$ & $0.88(0.09)$ \\
Dammak [9] & Vitallium beaded & & $0.68(0.09)$ \\
& $\quad$ porous surface & & $0.58(0.12)$ \\
Shirazi [31] & Titanium bead & & $0.60(0.11)$ \\
& Titanium fiber mesh & & $0.53(0.07)$ \\
Rancourt [30] & Sintered titanium beads & & $0.47(0.03)$ \\
& Titanium fiber mesh & & \\
Current study & Wave & $0.68(0.04)$ & \\
& Cubic & $0.63(0.03)$ & \\
& Ti plasma sprayed & $0.64(0.04)$ & \\
& $\quad$ coating & $0.49(0.06)$ & \\
& Sandblasted & &
\end{tabular}

Values are results and SD

roughness of the investigated surfaces. However, it appeared to be impossible to define the roughness of the new surface structures accurately, because of the three dimensional character of the new surface structures.

The friction coefficients of the new E-beam specimens were significantly higher compared to the sandblasted specimens and are in the same range of the titanium plasma sprayed control. Since implants with a titanium plasma sprayed surface have a high survival rate (indicating sufficient initial stability) [23], it can be deduced that the two new surface structures can also provide sufficient initial stability (albeit that this will also largely depend on prosthetic shape). Several other studies have reported the frictional properties of coatings for orthopedic implants (Table 2) [9, 27-31]. Although the values of the friction coefficient may depend to some extent on the testing method which vary amongst the different studies, comparison to our results indicate that the new surface structures result in relatively high friction coefficients.

In this study three different methods were used to quantify bone ingrowth; all have advantages and disadvantages with regard to the interpretation of the results. For example, bone ingrowth depth is restricted by pore depth and measuring direct bone-implant contact is the only method which enables comparison of porous and solid specimens.

The amount of direct bone-implant contact of the E-beam produced surface structures appeared to be comparable to the titanium plasma sprayed control. Hence, the new surface structures have the potential to be successful surface structures for orthopedic implants. 
The cubic structure showed greater bone ingrowth depth compared to the wave structure. On the contrary, the wave structure showed better results for bone area percentage. This difference in outcome can be explained by differences in the structure of the coating. The cubic specimen has a high porosity and large pores deep inside the core material, which were too deep for the bone to reach in the limited postoperative time ( 6 weeks) of the study. Consequently bone area percentage of the cubic specimen was less than the wave specimen.

Furthermore, the importance of pore size and porosity influencing bone ingrowth is supported by the differences in bone ingrowth between the 3-dimensional surface structures and the rough E-beam control. Although made of the same material and manufactured using the same methods, the bone ingrowth of the new surface structures (large pores, high porosity) was greater compared to the sandblasted (plain) E-beam control.

Although it is clear that pore size affects bone ingrowth, the optimal pore size has yet to be determined. Bobyn et al. [32] considered $100-400 \mu \mathrm{m}$ as the optimum pore size range for bone ingrowth, but revealed no significant differences in bone ingrowth between pore sizes in this range and larger pores at 12 weeks after implantation. Similar results were found by Fisher et al. [33]. Bobyn et al. [15] showed that the extent of ingrowth of implants with pores of $710 \mu \mathrm{m}$ was significantly greater compared to those with pores of $550 \mu \mathrm{m}$ at 4 and 16 weeks after implantation. This indicates that $400 \mu \mathrm{m}$ is not the maximum pore size to enhance bone ingrowth.

One setback of a larger pore size and a higher porosity is the length of time it takes for full integration of bone into the implant. Hing et al. [34] showed that the volume of bone ingrowth and the bone-implant contact of specimens with high porosities $(80 \%)$ increases gradually from 5,13 and 26 weeks after implantation. Bobyn et al. [15] demonstrated the same effect for pore sizes of 430 and $650 \mu \mathrm{m}$. These observations concur with the results of our study showing that the ultimate bone ingrowth is not accomplished for the new E-beam structures in the 6-week study period. Based on the current results bone ingrowth is likely to continue after the 6-week study period and will further anchor the implant to the bone. However, one can expect that ingrowth beyond a certain depth does not enhance the strength of the bone-implant interface, similar as seen for the cement-bone interface [35].

One of the few studies on E-beam engineered surfaces in the orthopedic literature is reported by Ponader et al. [22] They demonstrated a reduced proliferation of human fetal osteoblasts on porous E-beam produced surfaces, compared to smooth and unprocessed E-beam surfaces. However, in that same study, SEM analysis demonstrated that the cells attached and spread well on all surfaces [22]. This indicates that the E-beam produced material itself does not hamper cell viability and proliferation, but that this may be influenced by the micro and macro geometrical characteristics. In contrast to the study of Ponader et al. [22], the E-beam produced surface structures used in this study were superior compared to a sandblasted E-beam produced specimen.

Several explanations can clarify this difference; differences in finishing surface treatment, testing methods and surface characteristics. With respect to the finishing treatment, an additional step was added in the surface structures tested in this study. Before biological cleaning and sterilization a sandblasting step was performed in order to remove all residual powder particles. Regarding the method of testing, it is questionable whether the examination of pre-osteoblastic cell proliferation of Ponader et al. [22] provides valid data for bone ingrowth of prosthetic components. In this process stroma cells probably play a more prominent role [36]. Concerning surface characteristics, two out of the three surface structures tested by Ponader et al. [22] had smaller pores than the structures used in this study. The pore size of the third surface structure was comparable. Additionally, the porosity of these structures was lower as well. The superiority of our structures suggests that a large pore size and high porosity enhance bone ingrowth and support the importance of surface characteristics influencing bone ingrowth.

In conclusion, the newly developed surface structures engineered in this study provide sufficient friction at the bone-implant interface thus achieving initial stability. The ultimate bone ingrowth was not accomplished in this study, due to the high porosity and large average pore size of the new E-beam surface structures. However, the bone ingrowth into the new surface structures appears to be comparable to more conventionally made surfaces of clinically successful implants at 6 weeks after surgery.

The results of this study are promising as bone ingrowth is likely to continue after the 6 weeks allotted for this study. Testing of bone ingrowth for an extended period is necessary to support our hypothesis that the new surface structures can provide improved fixation properties compared to conventionally made surfaces.

Acknowledgments Eurocoating SpA, Trento, Italy, designed the new surface structures and supported this study. The authors thank Willem van de Wijdeven and Léon Driessen for their technical assistance and the staff of the central animal facility of the Radboud University Nijmegen for their assistance in the animal experiments. These experiments comply with the current laws of the Netherlands.

Open Access This article is distributed under the terms of the Creative Commons Attribution Noncommercial License which permits any noncommercial use, distribution, and reproduction in any medium, provided the original author(s) and source are credited. 


\section{References}

1. Mendenhall S (2004) Hip and knee implant review. Orthop Netw News 15:1-16

2. Eskelinen A, Remes V, Helenius I, Pulkkinen P, Nevalainen J, Paavolainen P (2006) Uncemented total hip arthroplasty for primary osteoarthritis in young patients: a mid-to long-term follow-up study from the Finnish Arthroplasty Register. Acta Orthop 77:5770

3. Karrholm J, Garellick G, Herberts P (2007) Annual report 2006. Swedish National Hip Arthroplasty Register

4. Ulrich SD, Seyler TM, Bennett D, Delanois RE, Saleh KJ, Thongtrangan I, Kuskowski M, Cheng EY, Sharkey PF, Parvizi J, Stiehl JB, Mont MA (2008) Total hip arthroplasties: what are the reasons for revision? Int Orthop 32:597-604

5. Sundfeldt M, Carlsson LV, Johansson CB, Thomsen P, Gretzer C (2006) Aseptic loosening, not only a question of wear: a review of different theories. Acta Orthop 77:177-197

6. McAuley JP, Szuszczewicz ES, Young A, Engh CA Sr (2004) Total hip arthroplasty in patients 50 years and younger. Clin Orthop Relat Res 418:119-125

7. Kurtz SM, Ong KL, Schmier J, Mowat F, Saleh K, Dybvik E, Karrholm J, Garellick G, Havelin LI, Furnes O, Malchau H, Lau E (2007) Future clinical and economic impact of revision total hip and knee arthroplasty. J Bone Jt Surg Am 89(Suppl 3):144-151

8. Ryd L, Albrektsson BE, Carlsson L, Dansgard F, Herberts P, Lindstrand A, Regner L, Toksvig-Larsen S (1995) Roentgen stereophotogrammetric analysis as a predictor of mechanical loosening of knee prostheses. J Bone Jt Surg Br 77:377-383

9. Dammak M, Shirazi-Adl A, Schwartz M Jr, Gustavson L (1997) Friction properties at the bone-metal interface: comparison of four different porous metal surfaces. J Biomed Mater Res 35:329-336

10. Geetha M, Singh AK, Asokamani R, Gogia AK (2009) Ti based biomaterials, the ultimate choice for orthopaedic implants-a review. Prog Mater Sci 54:397-425

11. Kienapfel H, Sprey C, Wilke A, Griss P (1999) Implant fixation by bone ingrowth. J Arthroplasty 14:355-368

12. Buser D, Schenk RK, Steinemann S, Fiorellini JP, Fox CH, Stich $H$ (1991) Influence of surface characteristics on bone integration of titanium implants. A histomorphometric study in miniature pigs. J Biomed Mater Res 25:889-902

13. Shalabi MM, Gortemaker A, Van't Hof MA, Jansen JA, Creugers $\mathrm{NH}$ (2006) Implant surface roughness and bone healing: a systematic review. J Dent Res 85:496-500

14. Petersilge WJ, D'Lima DD, Walker RH, Colwell CW Jr (1997) Prospective study of 100 consecutive Harris-Galante porous total hip arthroplasties. 4- to 8-year follow-up study. J Arthroplasty 12:185-193

15. Bobyn JD, Stackpool GJ, Hacking SA, Tanzer M, Krygier JJ (1999) Characteristics of bone ingrowth and interface mechanics of a new porous tantalum biomaterial. J Bone Jt Surg Br 81:907914

16. Otsuki B, Takemoto M, Fujibayashi S, Neo M, Kokubo T, Nakamura T (2006) Pore throat size and connectivity determine bone and tissue ingrowth into porous implants: three-dimensional micro-CT based structural analyses of porous bioactive titanium implants. Biomaterials 27:5892-5900

17. Karageorgiou V, Kaplan D (2005) Porosity of 3D biomaterial scaffolds and osteogenesis. Biomaterials 26:5474-5491

18. Jones JR, Ehrenfried LM, Hench LL (2006) Optimising bioactive glass scaffolds for bone tissue engineering. Biomaterials 27:964973
19. Heinl P, Rottmair A, Körner C, Singer R (2007) Cellular titanium by selective electron beam melting. Adv Eng Mater 9:360-364

20. Heinl P, Muller L, Korner C, Singer RF, Muller FA (2008) Cellular Ti-6Al-4V structures with interconnected macro porosity for bone implants fabricated by selective electron beam melting. Acta Biomater 4:1536-1544

21. Larson M, Lindhe U, Harrysson O (2003) Rapid manufacturing with electron beam melting (EBM) - a manufacturing revolution? In: Bourell D, Wood KL (eds) Solid freeform fabrication symposium proceedings. Austin, Texas, USA, pp 433-438

22. Ponader S, Vairaktaris E, Heinl P, Wilmowsky CV, Rottmair A, Korner C, Singer RF, Holst S, Schlegel KA, Neukam FW, Nkenke E (2008) Effects of topographical surface modifications of electron beam melted Ti-6Al-4 V titanium on human fetal osteoblasts. J Biomed Mater Res A 84:1111-1119

23. De Palma F, Erriquez A, Rossi R, Spinelli M (2007) Duofit total hip arthroplasty: a medium- to long-term clinical and radiographic evaluation. J Orthop Traumatol 8:117-122

24. Fellah BH, Gauthier O, Weiss P, Chappard D, Layrolle P (2008) Osteogenicity of biphasic calcium phosphate ceramics and bone autograft in a goat model. Biomaterials 29:1177-1188

25. Manders PJ, Wolke JG, Jansen JA (2006) Bone response adjacent to calcium phosphate electrostatic spray deposition coated implants: an experimental study in goats. Clin Oral Implants Res $17: 548-553$

26. Bordini B, Stea S, De CM, Strazzari S, Sasdelli A, Toni A (2007) Factors affecting aseptic loosening of 4750 total hip arthroplasties: multivariate survival analysis. BMC Musculoskelet Disord 8:69

27. Fitzpatrick D, Ahn P, Brown T, Poggie R (1997) Friction coefficients of porous tantalum and cancellous and cortical bone. In: Conference proceedings 21st annual meeting of the American Society of Biomechanics, Clemson, South Carolina, USA

28. Heiner AD, Brown TD (2007) Frictional coefficients of a new bone ingrowth structure. In: Transactions of the 53rd annual meeting of the Orthopaedic Research Society, San Diego, California, USA, pp 1623

29. Zhang Y (1999) Interfacial frictional behavior: cancellous bone, cortical bone, and a novel porous tantalum biomaterial. J Musculoskelet Res 3:245-251

30. Rancourt D, Shirazi-Adl A, Drouin G, Paiement G (1990) Friction properties of the interface between porous-surfaced metals and tibial cancellous bone. J Biomed Mater Res 24:1503-1519

31. Shirazi-Adl A, Dammak M, Paiement G (1993) Experimental determination of friction characteristics at the trabecular bone/porous-coated metal interface in cementless implants. J Biomed Mater Res 27:167-175

32. Bobyn JD, Pilliar RM, Cameron HU, Weatherly GC (1980) The optimum pore size for the fixation of porous-surfaced metal implants by the ingrowth of bone. Clin Orthop Relat Res 150:263-270

33. Fisher JP, Vehof JW, Dean D, van der Waerden JP, Holland TA, Mikos AG, Jansen JA (2002) Soft and hard tissue response to photocrosslinked poly(propylene fumarate) scaffolds in a rabbit model. J Biomed Mater Res 59:547-556

34. Hing KA, Best SM, Tanner KE, Bonfield W, Revell PA (2004) Mediation of bone ingrowth in porous hydroxyapatite bone graft substitutes. J Biomed Mater Res A 68:187-200

35. Majkowski RS, Bannister GC, Miles AW (1994) The effect of bleeding on the cement-bone interface. An experimental study. Clin Orthop Relat Res (299):293-297

36. Wall I, Donos N, Carlqvist K, Jones F, Brett P (2009) Modified titanium surfaces promote accelerated osteogenic differentiation of mesenchymal stromal cells in vitro. Bone 45:17-26 